\title{
Growth and Self-Assembly of One-Dimensional Magnetic Particles Chain of Polycrystalline Nickel by Chemical Reduction in the Presence of a Magnetic Field
}

\author{
XIE Sheng $\mathrm{Li}^{1, \mathrm{a}}$, GOU Jian Xia ${ }^{1, b}$ \\ ${ }^{1}$ Department of Chemistry and Chemical Engineer, Binzhou University, Shandong Binzhou China, \\ ashenglixie@126.com, bgoujianxia@163.com
}

Keywords: one-dimensional chain; nickel; magnetic field; reduction reaction

\begin{abstract}
One-dimensional magnetic particles chain of polycrystalline nickel was prepared by chemical reduction in the presence of a magnetic field. Based on solution reduction reaction of $\mathrm{Ni}^{2+}$, metallic nickel was deposited and synchronously self-assembled into chains under magnetic field. The results show that the chain of polycrystalline nickel was several microns in length and approximately $500 \mathrm{~nm}$ in diameter. The formation mechanism of the wires was studied by taking samples at different time in the formation process of the nickel wires. It is suggested that the magnetic field be an effective and tunable way to the fabrication of one-dimensional chains of magnetic materials.
\end{abstract}

\section{Introduction}

In recent years, one-dimensional wires have been becoming the focus of intense research due to their potential application, such as magnetic sensors, electro optical and sensing devices and the push to minimize of computational devices ${ }^{[1]}$. Magnetic metal materials such as $\mathrm{Fe}^{[2,3]}, \mathrm{Co}^{[4,5,6]}$, and $\mathrm{Ni}^{[7-12]}$ have been studied for many years. The wires composed of magnetic metal particles are drawing much attention. Many efforts have been concentrated on the manipulation of the magnetic metal materials using the magnetic field. It has been found that the magnetic field can significantly influence the properties of the magnetic materials ${ }^{[13,14]}$.

In this paper, the growth and assembly behavior of nickel magnetic nanocrystallites under an external $0.3 \mathrm{~T}$ magnetic field were studied. We report the synthesis of polycrystalline nickel wires without any template under magnetic field. In this method as soon as the polycrystalline Ni formed, it would be self-assembled into wires under magnetic field. Nickel would grow easily under appropriate supersaturation along the magnetic field into wires. The wires were nearly parallel because their axes were all parallel to the magnetic line of force.

\section{Methods}

In the experiment, all the reagents are analytical grade or higher. The experiment was carried out as the following steps. First, the solution containing $7.88 \mathrm{gL}^{-1} \mathrm{NiSO}_{4}, 12.72 \mathrm{gL}^{-1}$ potassium sodium tartrate and $5.0 \mathrm{gL}^{-1}$ sodium hydroxide was prepared. Second, put the solution in the symmetrical $0.3 \mathrm{~T}$ magnetic field and heat it up to $80^{\circ} \mathrm{C}$. Then the reducer hydrazine was added simultaneity with strongly stirring for a while. The color of the solution changed from grass-green to sea-blue. The reaction was beginning after 50min and stopped after $90 \mathrm{~min}$. Third, the nickel was washed with distilled water and ethanol for several times respectively. Last, the product was characterized by the scanning electron microscopy (SEM, LEO, 1530), the transmission electron microscopy(TEM, JEOL, Japan, Hitachi), selected area electron diffraction pattern(SAED, JEOL, Japan, Hitachi) and X-ray powder diffraction (XRD) with $\mathrm{Cu} \mathrm{K \alpha}$ radiation $\left(\lambda=1.54056 \times 10^{-10} \mathrm{~m}\right)$. Magnetic hysteresis loops were measured by a vibrating sample magnetometer (VSM, Model 7400, LakeShore).

The chemical reaction for the synthesis of nickel nanocrystallite can be expressed as below:

$$
2 \mathrm{Ni}^{2+}+\mathrm{N}_{2} \mathrm{H}_{4}+4 \mathrm{OH}^{-}=2 \mathrm{Ni} \downarrow+\mathrm{N}_{2} \uparrow+4 \mathrm{H}_{2} \mathrm{O}
$$




\section{Results and Discussion}

One drop of the suspension was carefully dipped on the silicon wafer in magnetic field for SEM observation. The image of the sample was shown in Figure 1 (a). The result agreed well with the anticipation that the wires patterned orderly on the silicon wafer. The wires were homogeneous in the diameter and length. The magnified image of the sample was shown in Figure 1 (b). The wire was about $500 \mathrm{~nm}$ in diameter and several microns in length. There were many acicular nickel nanocrystallites on the edge of the wires. It is reported that the acicular nickel nanocrystallites are single crystal ${ }^{[15]}$. So the formation of the acicular nickel nanocrystallites might be affected by the crystal nucleation interaction. The chemical composition of the wires was determined using the EDX. In the EDX spectrum of the wires (Figure1c) the peaks of Ni was only found on the silicon. So we can conclude that the wires were composed of nickel element only.

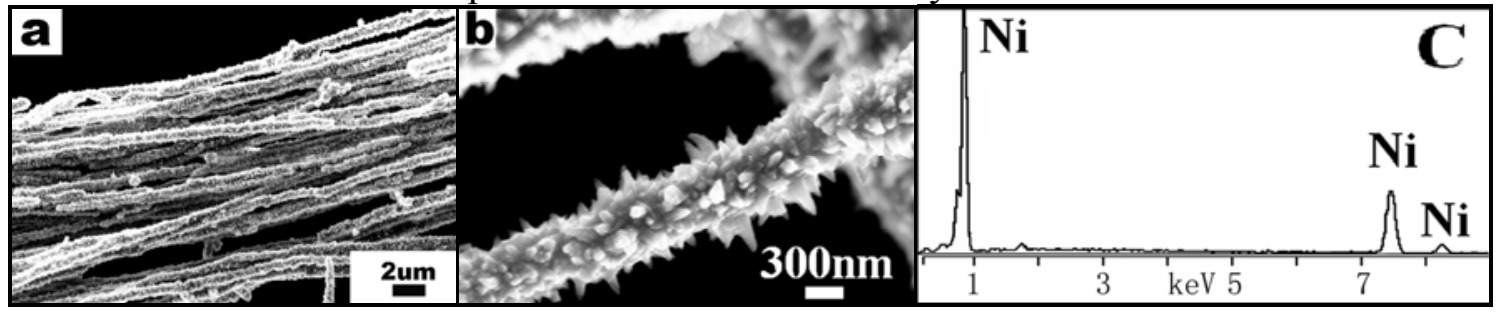

Figure1. (a) SEM image of the sample. (b) The magnified image of the sample. (c) EDX spectra of the sample.

The samples taking at different time in the formation of the nickel wires were observed by the TEM. The images were showed in Figure 2. (a) is the image of the neonatal sample . (b) is the image of a wire in process of formation. The wires are uniform and perfect. The wires' diameter and length is corresponding to the results of SEM. The SAED pattern taken on the surface of a wire is shown in the Figure $2 \mathrm{c}$. The diffraction spots correspond to the (111), (200), (220) and (311) diffraction planes of the nickel crystal. The TEM image (Figure 2) show that the crystallite of nickel formed at the beginning of the reaction then self-assembled into wires under the magnetic field. So the nickel changed from crystallite into polycrystalline.

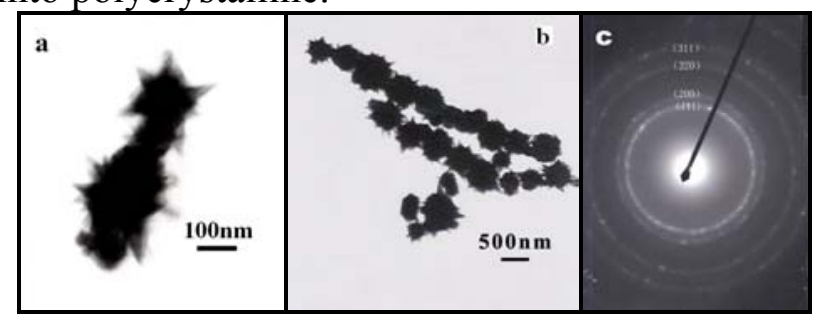

Figure2.TEM images of the sample. (a) (b) The image of the sample.(c) The SAED patterns taken on the surface of the sample.

At the beginning of the reaction, a number of the single nickel particles formed. Then they would self-assemble and aggregate into a big one and the big particle would magnetize in the magnetic field. With the proceeding of the reaction there would be more big particles formed. These big particles would array along the magnetic gradient. At the same time the single nickel particles would aggregate to the wires under the influence of magnetic dipoles interaction in the magnetic field. So the nickel wires formed. The nickel wires growth mechanism was shown in figure 3 .

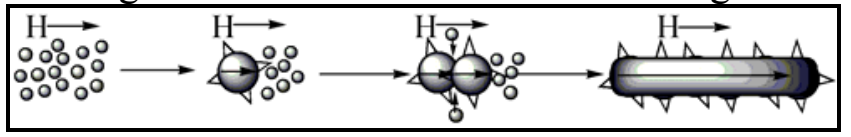

Figure3. A sketch map of the nickel wires growth mechanism.

The polycrystalline nature of the nickel was further confirmed by XRD pattern. The XRD pattern (Figure 4) shows sharp peaks corresponding to the (111), (200), (220) and (311) diffraction peaks of the metallic Ni crystal which agreed well with the SAED result. It can be well indexed with the reflections of face-centered cubic Ni. We calculated by the Scherrer's equation that the crystalline size is $11.2 \mathrm{~nm}$. The ratio among the intensity of (111), (200) and (220) diffraction peaks is 100:39:20 and the conventional value of (111), (200) and (220) is 100:42:21. The difference between our result 
and the conventional value can be ignored. So we can conclude that the formation of the wires isn't mainly dominated by any facets.

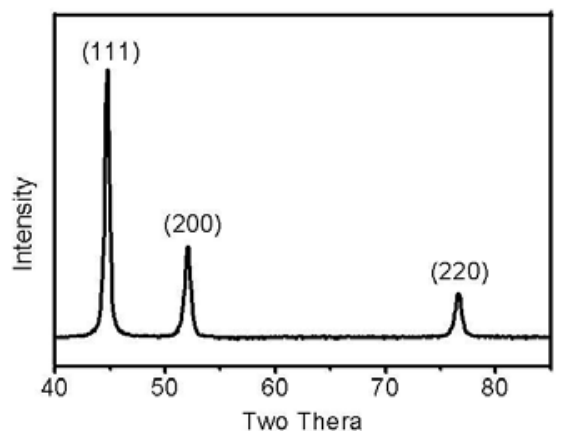

Figure4. XRD image of the sample.

The sample was prepared for the VSM using the same method as the preparation of sample for the SEM. The magnetic properties of the product were investigated by VSM (Figure 5). There are two ways to arrange the sample in the external magnetic field. One is the wires parallel to the magnetic field and the other is the wires perpendicular to the magnetic field. Figure 5.a presents the applied field parallel to the lengthwise direction of the self-assembled wires' structure. Its coercivity is $173.5 \mathrm{Oe}$, the saturation value of magnetization is $15.6 \mathrm{emu} / \mathrm{g}$ and $\mathrm{Mr}$ is $11.2 \mathrm{emu} / \mathrm{g}$. For comparison, b is perpendicular to the lengthwise direction of the wires. However its coercivity is $179.5 \mathrm{Oe}$. Ms is $12.2 \mathrm{emu} / \mathrm{g}$ and $\mathrm{Mr}$ is $3.8 \mathrm{emu} / \mathrm{g}$. Ms of a is higher than $\mathrm{b}$, but the coercivity of $\mathrm{a}$ is lower than $\mathrm{b}$. The $\mathrm{Mr}$ of $\mathrm{a}$ is 3 times of $\mathrm{b}$. It is suggested that the magnetic field would be a determinant to the wires' structure and magnetic properties.

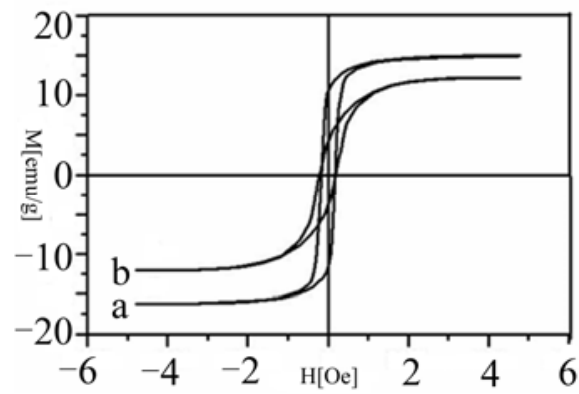

Figure5 hysteresis loops measured at room temperature a: The wires were parallel to the external magnetic field. b: The wires were perpendicularity. to the external magnetic field

\section{Summary}

In conclusion, polycrystalline nickel wires with several microns in length and about $500 \mathrm{~nm}$ in diameter were formed using the magnetic field. The mechanism of the wires' formation was studied by taking samples at the progress of the reaction. The magnetic field provided the attraction force among nickel crystallites to cause them to grow into wires. So the magnetic field played an important role in the formation of wires. The magnetic properties of wires were different when it was parallel to the magnetic field and perpendicular to the magnetic field, which might be associated with the special nanostructure that $\mathrm{Ni}$ nanocrystallites were arranged in polycrystalline wires acting as permanent magnetic dipoles. This method of formation of one-dimensional magnetic particle chain polycrystalline nickel was template-free and simple. The process could be used to fabricate large arrays of uniform wires of some magnetic materials and improve the magnetic properties of nanoscale magnetic materials.

\section{Acknowledgements}

This work was financially supported by the Foundation of Binzhou University for Excellent Youth Teachers (№BZXYQNLG200614, №BZXYQNLG 200612). 


\section{References}

[1] By Oded Rabin, Paul R. Herz, Yu-Ming Lin, Akintunde I. Akinwande, Setphen B.Cronin. Adv. Funct. Mater. Vol 13(2003), p. 631.

[2] de Caro. D, Ely, T. O, Mari. A, Chaudret, B. Snoeck. E, Respaud. M, Broto. J.-M.Fert. A. Chem. Mater. Vol 8(1996), p. 1987.

[3] Park, S.-J, Kim, S, Lee, S, Khim. Z.G, Char. K, Hyeon. T. J. Am. Chem. Soc. Vol 122(2000), p.8581.

[4] Gambardella. P, Ruspoi. S, Veronese. M, Dhesi. S, Grazioli. C, Dallmeyer. A, Cabria. I, Zeller.R, Dederichs. P. H, Kern. K, Carbone. C, Brune. H, Science. Vol 300(2003), p.1130.

[5] Osuna J, de Caro D, Amiens C, Chaudret B, Snoeck E.Respaud. M, Broto.J.-M, Fert. A. J. Phys. Chem. Vol100(1996), p.14571.

[6] Dinega. D. P, Bawendi. M.G, Angew. Chem. Int. Ed. Engl. Vol38(1999), p. 1788

[7] Cordente, N. O., Respaud, M.; Senocq, F.,Casanove, M.-J., Amiens, C., Chaudret, B. Nano Lett. Vol 1(2001), p.565.

[8] Hseng-Hsueng Lee, H. T. Kuo, Kan-Sen Chou. J. Chin. Inst. Chem. Engrs. Vol 34(2003), p.327. [9] PENG Mei-xun, SHEN Xiang-qian, WANG Ling-sen. Journal of Central South University of Technology, 2005, Vol 12(1)(2005), p.5.

[10] PENG Mei-xun, SHEN Xiang-qian. Journal of Central South University of Technology. Vol 14(3) (2007), p.310.

[11] SONG Quan-sheng, TANG Zhi-yuan, GUO He-tong, et al. J Power Sources. Vol 112(2) (2002), p. 428.

[12] Delahay Ye-vidal, Beaudoin B, Sac-Epee N. Solid State Ionics, Vol 84(4) (1996), p. 239.

[13] Fulmer. P, Manivel Raja. M, Manthiram. Chem. Mater. Vol 13(2001 2160.

[14] Dunin-Borkowski. R. E, McCartney. M. R, Frankel. R. B, Bazylinski. D. A. Science. Vol 282(1998), p.1868

[15] NIU He-lin., CHEN Qian-wang., NING Min., JIA Yao-shun., WANG Xiao-juan. J. Phys. Chem. B. Vol 108(2004), p.3996. 